\title{
Domino Exclusion Problem
}

\author{
Andrejs CIBULIS ${ }^{1}$, Walter TRUMP ${ }^{2}$ \\ ${ }^{1}$ Institute of Mathematics and Computer Science, University of Latvia, \\ Raina blvd. 29, Riga, LV-1459, Latvia \\ ${ }^{2}$ Retired from: Gymnasium Stein, Faber-Castell-Allee 10, 90547 Stein, Germany \\ andrejs.cibulis@lu.lv, w@trump.de
}

\begin{abstract}
The classic domino exclusion problem consists of finding minimum number $d(n)$ of dominoes on an $n \times n$ chessboard to prevent placement of another domino. This sequence of minimum numbers is discussed under A280984 at the On-Line Encyclopedia of Integer Sequences. With new theoretical insights and a specially designed computer program we were able to expand the sequence from $n=18$ to $n=33$. New upper bounds of $d(n)$ thought to be sharp have been obtained. The article also discusses the rectangle-free minimal domino packings. Small 3 -dimensional grid squares up to $n=6$ have been analysed.
\end{abstract}

Keywords: computer-assisted proof, domino, estimate, grid rectangle, matchstick, upper bound.

\section{Introduction}

During the Covid-19 pandemic, the first author was writing a book on recreational mathematics. In one chapter, both old and new tasks about matchsticks were collected. In relation to the number 19 , the following task was devised: "To create a vaccine that prevents Covid-19 virus from multiplying, it is necessary to colour a minimum number of unit edges of the $19 \times 19$ cell square so that each uncoloured edge has at least one point of contact with the coloured edge." As it turned out later this rather difficult task had far-reaching consequences. Solving this problem for small matchstick squares $n \times n$ the following sequence of minimum numbers was obtained, see Figure 1:

$$
2,3,6,9,12,17, \ldots
$$

Then, looking at The On-Line Encyclopedia of Integer Sequences (Shepard, 2017), it was understood that this matchstick problem is equivalent to the domino exclusion problem studied earlier in (Gyárfás et al., 1988). Domino exclusion problem consists of finding minimum number of dominoes on an $n \times n$ board to prevent placement of another domino, see Figure 2 as a transformation of Figure 1 in domino terminology.
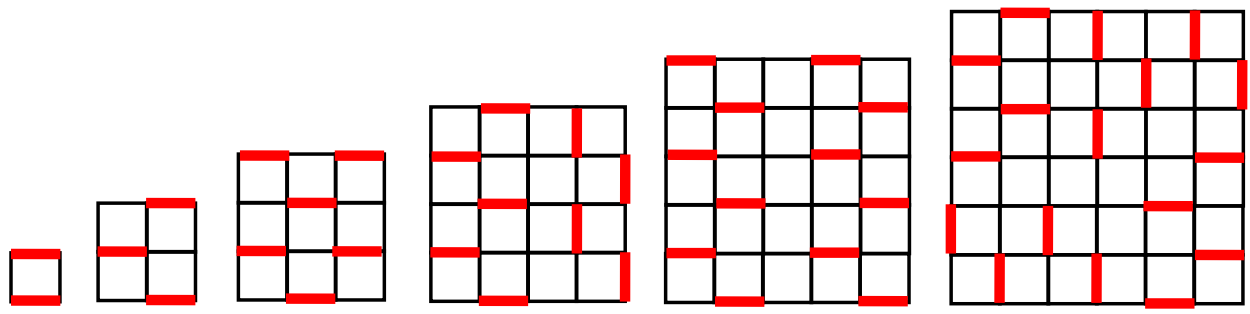

Figure 1. Minimum number of coloured sticks 

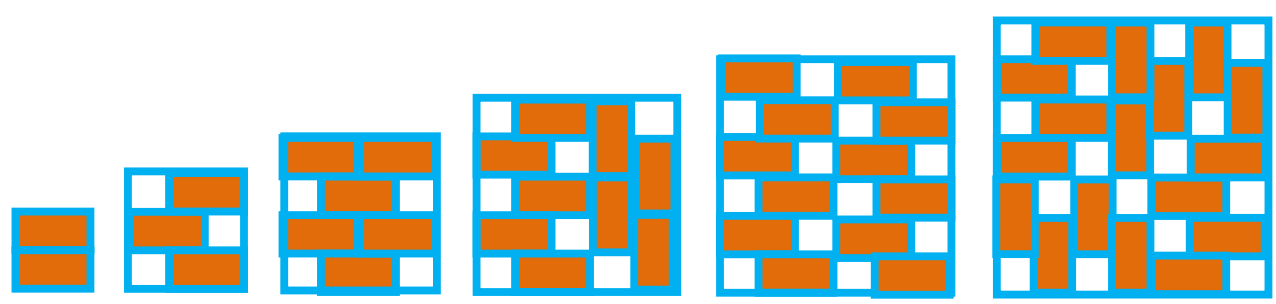

Figure 2. Minimum number of dominoes

A third way to visualize the problem and not to draw unpainted square edges at all is to use graphs, see Figures $3-4$.

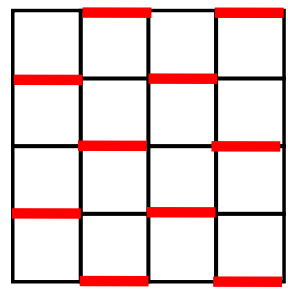

Figure 3. Non minimal arrangement with 10 sticks

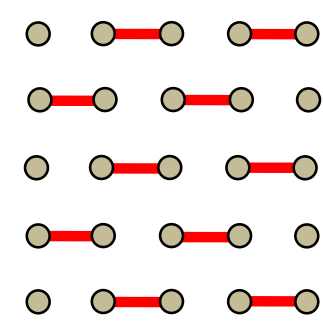

Figure 4. Interpretation by graph

As far as we know, the first book in which one can find the tasks of excluding shapes (namely, pentominoes by monominoes on a chessboard $8 \times 8$ ) is the Golomb's classic book (Golomb, 1994). Exclusion problems in other areas (graph theory, statistical physics, percolation theory) may have to do with the following concepts: matching, minimum dominating sets, domination number, square grid graphs, an edge cover, dimmers, and others (Alanko et al., 2011), (Korte and Vygen, 2018).

The second author developed an efficient algorithm that allowed new progress in both the domino exclusion problem and its generalizations to $n$-dimensional grids.

The article uses generally accepted terms in mathematics:

$$
\lceil x\rceil=\min \{n \in Z, n \geq x\},\lfloor x\rfloor=\max \{n \in Z, n \leq x\}
$$

are the so-called ceiling and floor functions of $x$, respectively. 


\section{Grid squares and rectangles}

Let $[m \times n]$ be a grid rectangle consisting of $m$ rows and $n$ columns of points (dots, vertices, grid meshes), and let $D=D(m, n)$ be the minimum number of dominoes (edges isolating grid points) for which a packing exists. A domino packing is an arrangement of dominoes on a given board (here on a grid rectangle) to prevent placement of another domino. The following numbers are of particular importance in future estimates:

$$
d(n):=D(n, n), D_{0}(m, n):=\left\lceil\frac{m n}{3}\right\rceil, m, n \geq 2, d_{0}(n):=D_{0}(n, n)
$$

A point is isolated if all its neighbours are connected by edges. Note that minimizing the number of edges or maximizing the number of isolated points or holes $H=H(m, n)$ are equivalent problems. Clearly that

$$
H+2 D=m n
$$

\subsection{Estimates}

Two important theorems are proved in this section. As a consequence of the first theorem, the following nice estimate is obtained:

$$
D(m, n) \geq\left\lceil\frac{m n}{3}\right\rceil, m, n \geq 2 .
$$

Both old and new (updated) information one can found in (Kagey, 2019): "Fifteen terms are known, and a few folks have conjectured that

$$
A 2808984(n)=\left\lceil\frac{n^{2}}{3}\right\rceil \text { for } n>1 .
$$

Walter Trump has just added the terms $19-33$ of the sequence (with $d(19)=122=\lceil 19 \cdot 19 / 3\rceil+1$ with some examples of optimal solutions and announced an effective algorithm for finding the optimal solutions. /Jun 14 at 20:30/"

The sequence of the left hand side of (3) here means the numbers $d(n)$, and since $d(19) \neq d_{0}(19)$, the conjecture (3) is generally incorrect. Here, ironically, numerologists should have known that Covid-19 number breaks for the first time this beautiful formula. Life is not so simple and we have to look for a new formula.

Such estimates of $d(n)$ are given in (Gyárfás et al., 1988): 


$$
d(n) \geq\left\lceil\frac{n^{2}}{3}\right\rceil, \quad d(n) \leq \frac{n^{2}}{3}+\frac{n}{12}+1
$$

"If $n$ is large and $n=3 k \pm 1$ then $d(n) \geq \frac{n^{2}}{3}+\frac{n}{111}$." (It is not specified how large).

Remark 1. $H \leq D+1$ is valid for strings (rectangles with one row or one column). Equality can only exist if the number of grid points is $n=3 k+1$, see Figure 5 with $H=4$ and $D=3$.

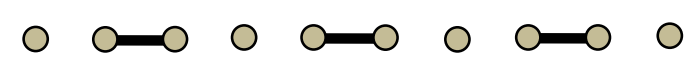

Figure 5. Domino arrangement with $H=D+1$

For rectangles, a stronger estimate $H \leq D$ can be proved (Theorem 1). The proof uses the same basic ideas as in the article (Gyárfás et al., 1988) but the presentation is shorter and quite different.

Theorem 1. $H(m, n) \leq D(m, n), m, n \geq 2$.

Proof. Let us use the following notations:

$B$ - set of boundary points of $[m \times n]$ rectangle (points in the 1 st or last row, or column)

$D, H$ - the number of dominoes, holes respectively,

$D_{B}, H_{B}$ - the number of dominoes, holes that are incident (have contact, touch) to $B$,

$D_{j}, H_{j}, j=1,2,3,4-$ the number of dominoes, respectively holes incident to the $j$-th

side of the rectangle, see Figure 6 where

$D_{1}=D_{3}=2, D_{2}=D_{4}=2, H_{1}=H_{3}=3, H_{2}=H_{4}=3$,

$T_{j}:=D-D_{j}+H_{2+j}-H, H_{5}:=H_{1}, H_{6}:=H_{2}, j=1,2,3,4$,

$T:=T_{1}+T_{2}+T_{3}+T_{4}$.

Such equalities:

$$
D=D_{j}+H-H_{2+j}+T_{j}, j=1,2,3,4,
$$

follow immediately from the notations, but their essence, salt, is in their geometric interpretation. Let us interpret (4), e.g. for $j=1$. The difference $H-H_{3}$ means the number of holes in the rectangle, except for the last row. Each such hole has a domino below it. It is important that each hole has its own corresponding domino. $T_{1}$ is the number of dominoes not yet counted. $T_{1}$ describes (redundant) dominoes exactly above which there are no holes and which do not belong to $D_{1}$. Figure 7 shows several domino 
arrangements in the form of tetrominoes I, L, and $\mathrm{O}$ which yield $T>0$. (Here is a motivation for choosing the symbol $T$ - tetromino.)

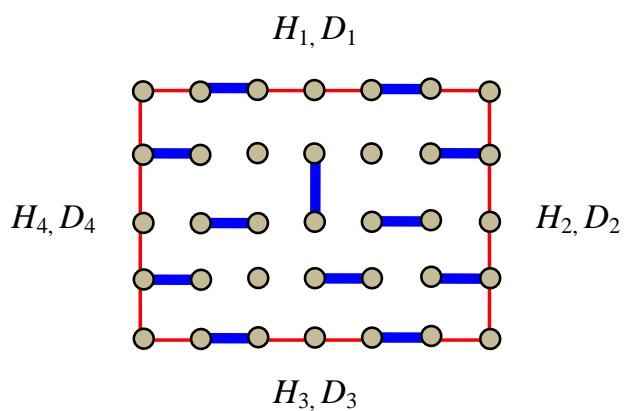

Figure 6. Example of $[5 \times 7]$ packing

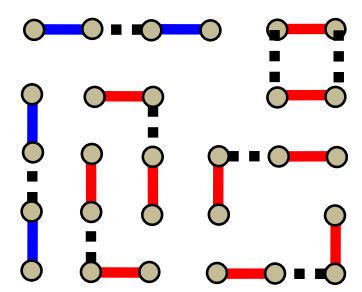

Figure 7. Arangements with $T>0$

Without loss of generality we can assume that $H_{1} \geq H_{3}$. Since $H_{1} \leq D_{1}+1$ then (4) immediately yields:

$$
D=D_{1}+H-H_{3}+T_{1} \geq H+D_{1}-H_{1}+T_{1} \geq H+T_{1}-1 .
$$

From here, $D \geq H$, if $H_{1} \leq D_{1}$. Due to (1): if $H=D+1$ then

$$
3 D+1=m n \Rightarrow m n=1(\bmod 3) \text {. }
$$

Thus, it remains to examine only those rectangles for which

$$
T_{j}=0, H_{j}=D_{j}+1, j=1,2,3,4, H=D+1, m n=1(\bmod 3) .
$$

Assume $R$ is the smallest rectangle that satisfies (5). The number of $B$ points is an even number, so they can be grouped in pairs. Since at least one point in each pair is the domino endpoint, then $H_{B} \leq D_{B}$. If $B$ would contain only dominoes with both endpoints inside $B$, then after discarding $B$ we would get a new rectangle smaller than $R$ with $H \geq D+1$ (and since $H>D+1$ is not possible then $H=D+1$ ), which contradicts the assumption. So there is a domino that has only one endpoint in $B$. Without loss of generality, we can assume that this is the horizontal domino with one point in the first column: $(i, 1), i \neq 1, n$, see Figure 8 . Then the points $(i \pm 1,1)$ are holes (otherwise the condition $H_{4}=D_{4}+1$ would not be fulfilled), but their adjacent points $(i \pm 1,2)$ are endpoints of horizontal dominoes. A vertically placed domino in points $(i \pm 1,2)$ formed L-tetromino arrangement (Figure 7) giving $T>0$. Since the number of columns 3 is not valid and the point $(i, 3)$ is a hole (otherwise $T>0)$ the points $(i, 4)$ and $(i, 5)$ are joined by domino. Points $(i \pm 1,4)$ are holes (otherwise $T>0$ ). Thus only the arrangements shown in Figure 9 is permissible. Since the process is not complete in the column $m \neq 3 k$, the theorem is proved. 


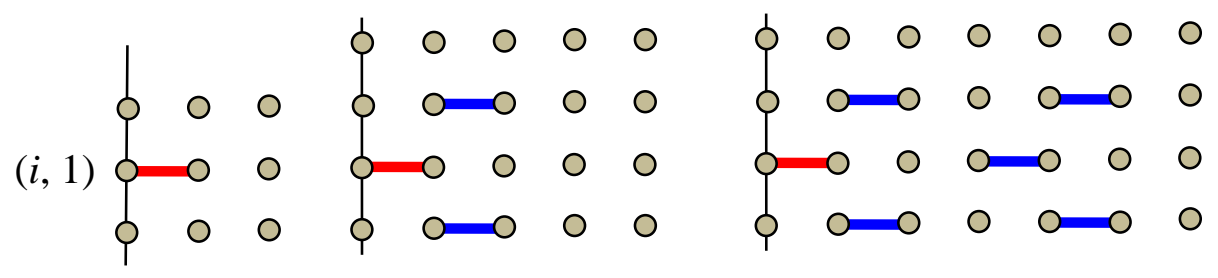

Figure 8.

Figure 9. Periodic arrangements

Remark 2. With this proof technique, an estimate $H \leq D$ can also be obtained for 3-dimensional grid rectangles.

Corollary. $D(m, n) \geq D_{0}(m, n)$, see (2).

Proof: $(H+2 D=m n, H \leq D) \Rightarrow 3 D \geq m n \Rightarrow D \geq \frac{m n}{3} \Rightarrow D \geq\left\lceil\frac{m n}{3}\right\rceil$ because $D$ is an integer.

Theorem 2. The following estimates are valid:

$$
\begin{gathered}
d(n) \leq d_{0}(n), n=3 k, \\
d(n) \leq d_{0}(n)+\left\lfloor\frac{n-3}{15}\right\rfloor, n=3 k+1, \\
d(n) \leq d_{0}(n)+\left\lfloor\frac{n}{21}\right\rfloor, n=3 k+2 .
\end{gathered}
$$

\section{Proof}

1. The case $n=3 k$, estimate (6), is trivial in the sense that minimal arrangements (solutions) also for $[m \times n]$ rectangles one can obtain using an elementary pattern shown in Figure 10. Copies of [ $m \times 3]$ can be added to each other as many times as needed. Moreover, it is important that the additive property holds:

$$
D_{0}\left(m_{1}+m_{2}, 3 k\right)=D_{0}\left(m_{1}, 3 k\right)+D_{0}\left(m_{2}, 3 k\right) .
$$




\begin{tabular}{|lll|lll|lll|}
\hline 0 & 0 & 0 & 0 & 0 & 0 & 0 & 0 & 0 \\
\hline 0 & 0 & 0 & 0 & 0 & 0 & 0 & 0 & 0 \\
\hline 0 & 0 & 0 & 0 & 0 & 0 & 0 & 0 & 0 \\
\hline 0 & 0 & 0 & 0 & 0 & 0 & 0 & 0 & 0 \\
\hline 0 & 0 & 0 & 0 & 0 & 0 & 0 & 0 & 0 \\
\hline
\end{tabular}

Figure 10. Periodic arrangement

2. Case $n=3 k+1$ is the most difficult to prove.

Let us compose the $[n \times n]$ square from elementary blocks: $[1 \times n]$ and $[4 \times n]$ rectangles, see Figure 11 with $n=4,7,10$.

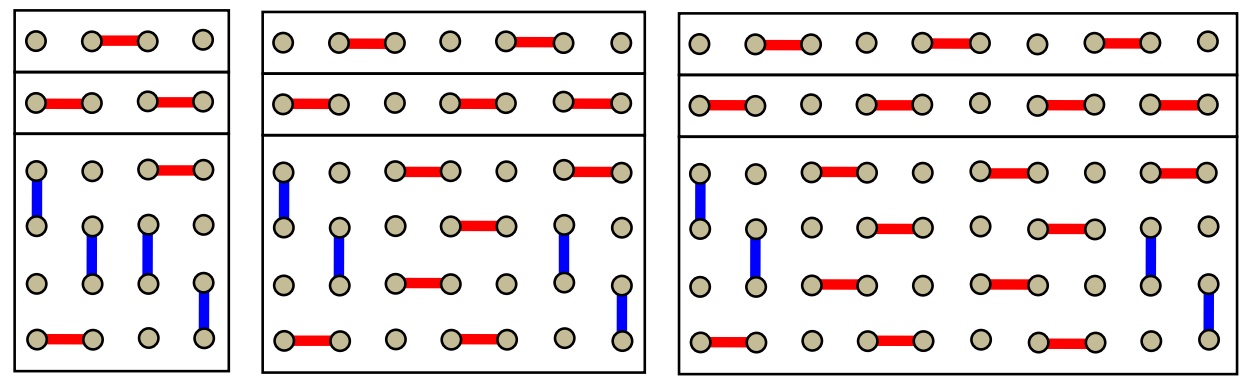

Figure 11. Elementary blocks for forming squares with $n=3 k+1$

It is clear that each such block can be stretched to a length $n=3 k+1$. Suppose we have $x$ rectangles $[1 \times n]$ and $y$ rectangles $[4 \times n]$. Then $x+4 y=n$. There are two types of $[1 \times n]$ rectangles with the following number of coloured edges: $\frac{n-1}{3}$, and $\frac{n+2}{3}$, respectively. If these two types of rectangles are adjacent and form $[2 \times n]$ then

$$
D(2, n)=\frac{2 n+1}{3}=2 k+1 \text {. }
$$

The number of coloured edges for $[4 \times n]$ rectangles is $D(4, n)=\left\lceil\frac{4 n}{3}\right\rceil$. Note that two $[4 \times n]$ rectangles are not adjacent, because in that case we would not get the minimal arrangement:

$$
2\left\lceil\frac{4(3 k+1)}{3}\right\rceil=8 k+4>\left\lceil\frac{8(3 k+1)}{3}\right\rceil=8 k+3 .
$$


Let's choose the following number of $[4 \times n]$ rectangles: $y=\left\lfloor\frac{n+1}{5}\right\rfloor$. The motivation for such a choice is that length 4 is followed by length 1 and we want to use the maximum number of such rectangles. For example, if $n=19$, then $y=4$, and partition of 19 is as follows: $19=4+1+4+1+4+1+4=4 \cdot 4+3 \cdot 1$. For simplification purposes, let us use the following elementary calculations:

$$
\begin{gathered}
\left\lceil\frac{4 n}{3}\right\rceil=\left\lceil\frac{4(3 k+1)}{3}\right\rceil=4 k+2 ; \\
\left\lceil\frac{n^{2}}{3}\right\rfloor+\left\lfloor\frac{n-3}{15}\right\rfloor=3 k^{2}+2 k+1+\left\lfloor\frac{3 k-2}{15}\right\rfloor ; n k=3 k^{2}+k .
\end{gathered}
$$

2a. If all $[1 \times n]$ rectangles contain $k$ coloured edges, then (since $x+4 y=n$ )

$$
\begin{gathered}
d(n) \leq y(4 k+2)+x k=k n+2 y \leq\left\lceil\frac{n^{2}}{3}\right\rceil+\left\lfloor\frac{n-3}{15}\right\rfloor \Leftrightarrow \\
2\left\lfloor\frac{3 k+2}{5}\right\rfloor \leq k+1+\left\lfloor\frac{3 k-2}{15}\right\rfloor .
\end{gathered}
$$

The correctness of this inequality can be easily proved by taking $k=5 j+r$ and checking the five values of remainder $r$.

2b. If all $[1 \times n]$ rectangles except one contain $k$ coloured edges then, see (9),

$$
d(n) \leq y(4 k+2)+(x-2) k+2 k+1=y(4 k+2)+x k+1,
$$

and instead of (10) we now have the inequality

$$
2\left\lfloor\frac{3 k+2}{5}\right\rfloor \leq k+\left\lfloor\frac{3 k-2}{15}\right\rfloor
$$

This inequality for arbitrary $k$ is not correct at all, for example, $k=3$. But here there is a subtle nuance, namely, the inequality does not have to be checked for all $k$, but only for those $k$ for which $n=3 k+1$ does not fit in the case $2 \mathbf{a}$. Note that only such $k$ values need to be checked: $k=5 j+r$, where $r=2$ or 4 . It is easy to prove that the inequality (11) for both values of $r$ transforms into equality.

3. Case $n=3 k+2$. Let us compose the $[n \times n]$ grid square from $x$ rectangles $[1 \times n]$ and $y$ rectangles $[3 \times n]$ with

$$
D(1, n)=\frac{n+1}{3}, D(3, n)=n \text {. }
$$


See Figure 12 with such elementary rectangles for $n=20$. Let's choose the following number of $[1 \times n]$ rectangles: $x=2+3\left\lfloor\frac{k}{7}\right\rfloor$.

Since $x+3 y=n$, then

$$
\begin{aligned}
d(n) & \leq \frac{x(n+1)}{3}+y n=\frac{x n+x}{3}+\frac{(n-x) n}{3}=\frac{x}{3}+\frac{n^{2}}{3}=\frac{2}{3}+\left\lfloor\frac{k}{7}\right\rfloor+\frac{n^{2}}{3}= \\
& =\frac{2+n^{2}}{3}+\left\lfloor\frac{k}{7}\right\rfloor \leq\left\lceil\frac{n^{2}}{3}\right\rfloor+\left\lfloor\frac{3 k+2}{21}\right\rfloor \Leftrightarrow\left\lfloor\frac{k}{7}\right\rfloor \leq\left\lfloor\frac{k}{7}+\frac{2}{21}\right\rfloor .
\end{aligned}
$$

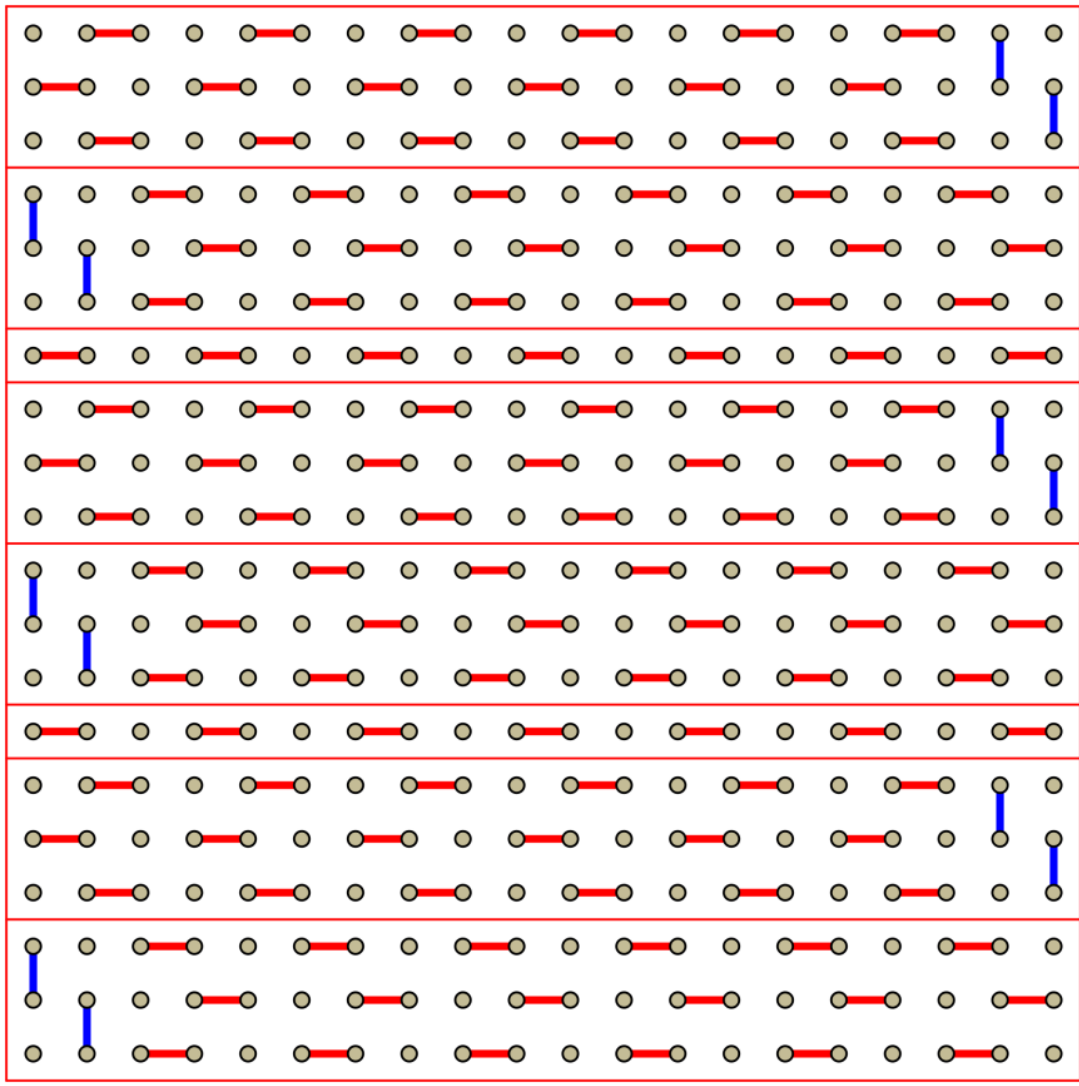

Figure 12. Minimal arrangement, $d(20)=134$ 


\subsection{Rectangles with $D(m, n)=D_{0}(m, n)$}

We have previously found that the smallest square for which equality (12) does not hold is $[19 \times 19]$. In this section we will look for the smallest rectangle for which the analogue of equality (12), i.e. (13) is no longer valid.

$$
\begin{gathered}
D(n, n)=D_{0}(n, n)=d_{0}(n)=\left\lceil\frac{n^{2}}{3}\right\rceil \\
D(m, n)=D_{0}(n, m)=\left\lceil\frac{n m}{3}\right\rceil .
\end{gathered}
$$

Theorem 3. $D(m, n)=\left\lceil\frac{m n}{3}\right\rceil$ for $2 \leq m \leq 13, n \geq 2$.

Proof. According to (2) it suffices to show solutions (arrangements) with the specified number $D_{0}(n, m)$ of dominoes. Such solutions are easy to find for small $m$. For $m=2$, see Figure 13, and for $m=3 k$, see the periodic arrangement shown in Figure 10.
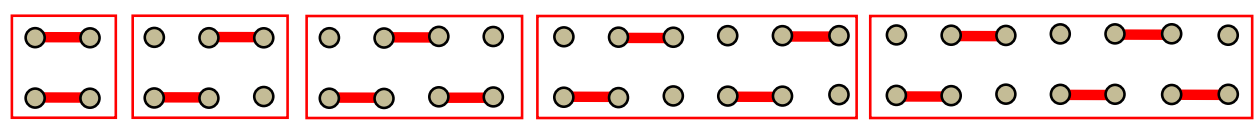

Figure 13. Minimal arrangements for $m=2$

The fact that the presented solutions contain the required number of dominoes can be easily verified using the property:

$$
D_{0}(m, j+3 k)=D_{0}(m, j)+D_{0}(j, 3 k) .
$$

Periodically added rectangles is also of use for $m=4$, and $m=5$, see Figure 14 . By repeating $[4 \times 3]$ and $[5 \times 3]$ rectangles the required number of times, we can get $[4 \times j+3 k],[5 \times j+3 k], j=1,2,3$, i.e. all necessary rectangles with $m=4$ and $m=5$.

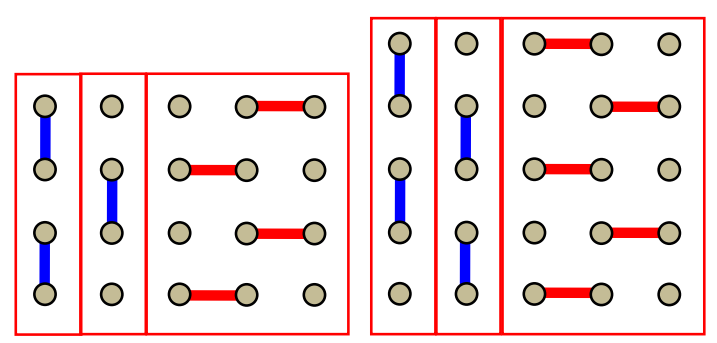

Figure 14. Minimal arrangements for $m=4$ and $m=5$ 
Now let's use a more advanced idea: in the role of elementary rectangles, let's take appropriately selected blocks that are periodically added. For $m=13$ the minimal arrangements are shown in Figure 15. The key to the proof is now a periodically movable string (a block of red edges), which we move by three units to obtain all the required rectangles. From here, removing the first two rows, we easily get the minimal arrangements for rectangles with $m=11$ rows. Similarly, removing the appropriate number of rows we will obtain minimal arrangements for the other required values for $m$ ( $m=10,8$, and 7). To avoid ambiguity, let us clarify that in the case $n=3 k+5$, after removing the first two rows, the vertical domino is shifted down one unit, and after removing the first 5 rows, the vertical domino is replaced by a horizontal one. Theorem is proved.

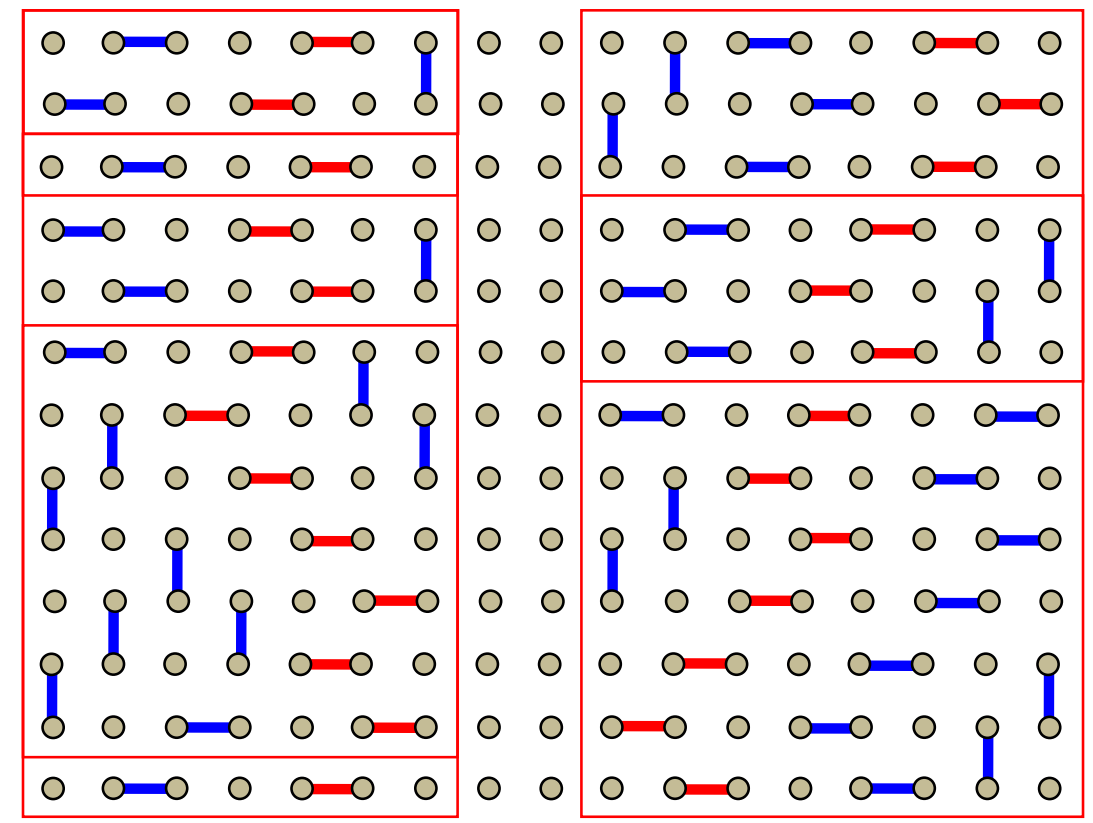

Figure 15. Minimal arrangements for $m=13, n=3 k+4, n=3 k+5$

By computer assisted-proof it is stated that $[14 \times 16]$ is the smallest rectangle for which the formula (13) is no longer valid. The smallest rectangle of type $[m \times(m+1)]$, for which the formula is not valid, is $[16 \times 17]$. As for the rectangles $[14 \times n], n=2(\bmod 3)$, this formula is again correct, see Figure 16 . 

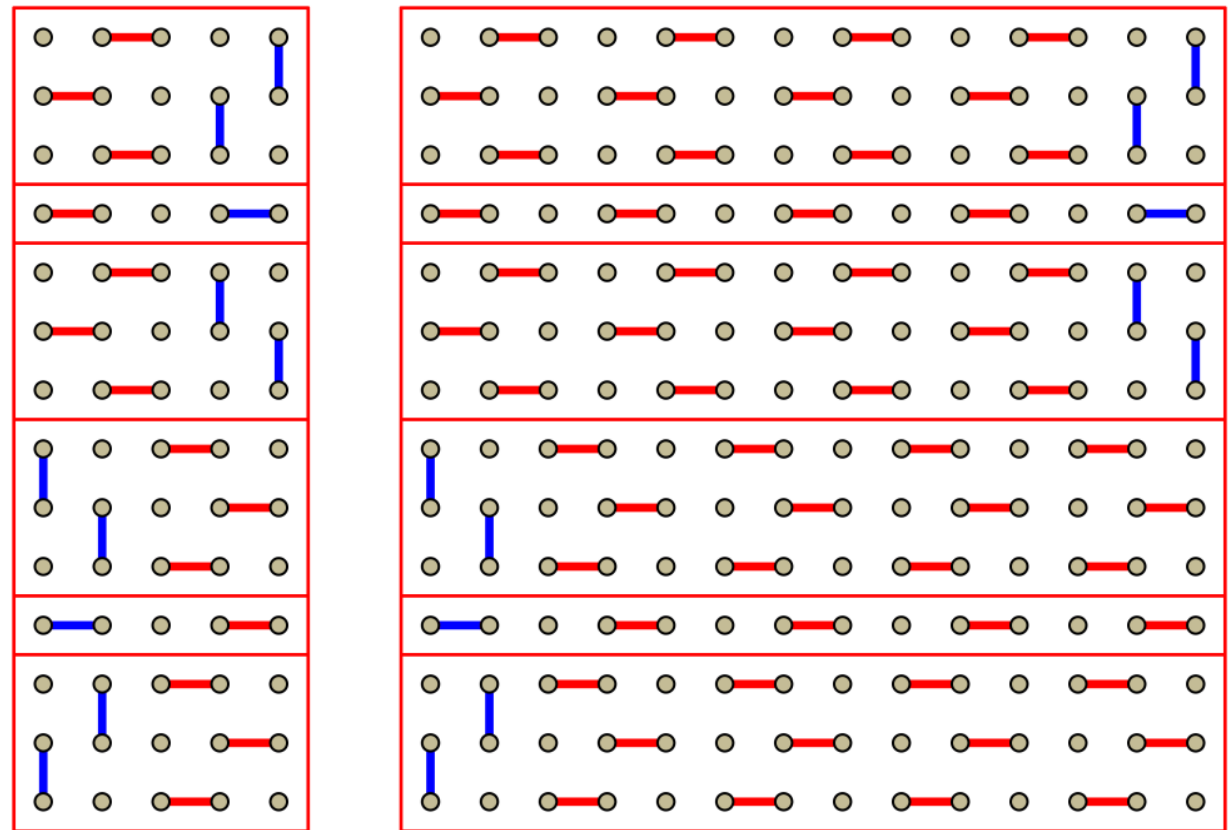

Figure 16. Minimal arrangements for $m=14, n=2+3 k$

Remark 3. Analogous estimates for rectangles can also be obtained by a similar proof technique (as in Theorem 1). A more complicated estimate is for rectangles whose edge lengths divided by three give different remainders:

$$
D(m, n) \leq\left\lceil\frac{m n}{3}\right\rceil+\min \left\{\left\lfloor\frac{m+3}{15}\right\rfloor,\left\lfloor\frac{n+6}{21}\right\rfloor\right\}, m=2(\bmod 3), n=1(\bmod 3)
$$

Remark 4. As a further study, we propose the following hypothesis:

$$
d(n)=\left\{\begin{array}{l}
\left\lceil\frac{n^{2}}{3}\right\rceil+\left\lfloor\frac{n-3}{15}\right\rfloor, n=3 k+1, \\
\left\lceil\frac{n^{2}}{3}\right\rfloor+\left\lfloor\frac{n}{21}\right\rfloor, n=3 k+2 .
\end{array}\right.
$$




\subsection{Rectangle-free packings}

In the previous section, the partition of squares in rectangles was crucial to prove the theorems. Let us now consider the question of the existence of minimal packings which cannot be divided into smaller rectangles. Such packings will be called rectanglefree packings (arrangements, solutions). The smallest square for which a rectangle-free packing exists is the $[5 \times 5]$ square, the next such squares are $[8 \times 8],[10 \times 10],[11 \times 11]$

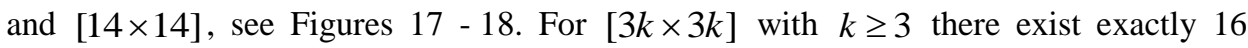
different packings if reflected and rotated solutions were count. But up to symmetry there are only 3 packings, see Figure 19. No packing is rectangle-free.

With the help of a computer program, it has been found that rectangle-free packings with $d(n)=d_{0}(n)$ is a rarity in general, the largest square for which such a solution still exists is $[14 \times 14]$. This is an unexpected result, at least for the first time, because larger squares no longer have this type of solution. For rectangles (unlike squares) the number of rectangle-free packings with $d(m, n)=d_{0}(m, n)$ is not finite, see, e.g. Figure 20 obtained from the $[5 \times 5]$ square repeating the red fragment. The fact that there is no a rectangle-free packing for $[n \times n]$ square does not mean that there is no a rectangle-free packing for $[m \times n]$ rectangle. See Figure 21 as an example.

Developing the idea of periodicity in two directions, we manage to find rectanglefree packings of squares with $d(n)$ satisfying (7) or (8) for arbitrarily large rectangles, see Figure 22. So far rectangle-free minimal packings with $d(n)=d_{0}(n)+1$ are known for $n=19,22,23,26$. See Figure 23 for $n=26$.

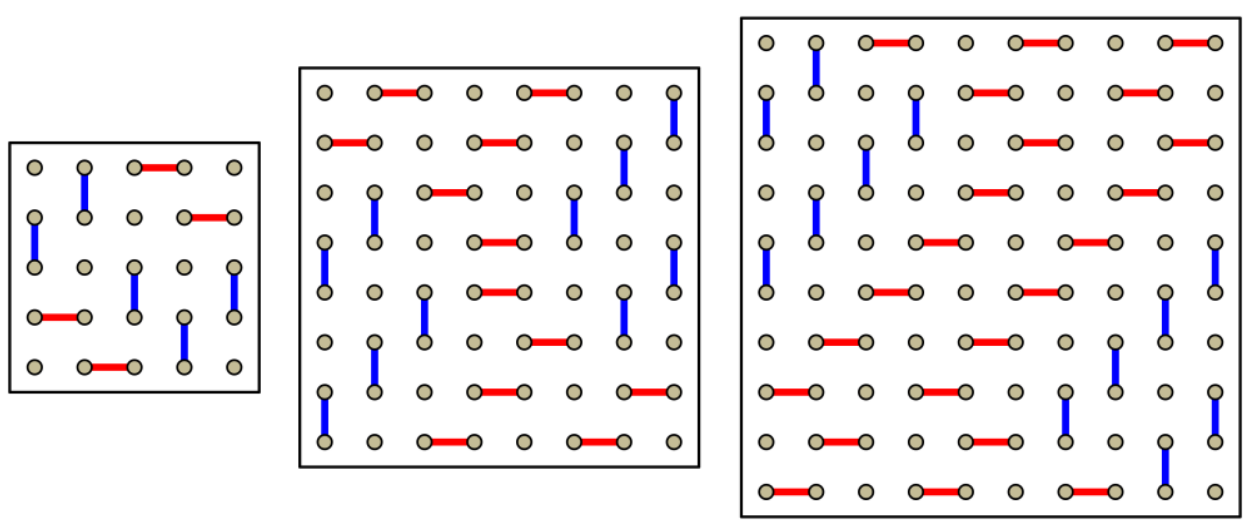

Figure 17. Rectangle-free packings: $d(5)=9, d(8)=22, d(10)=34$ 
Figure 18. Rectangle-free packings, $d(11)=41, d(14)=66$

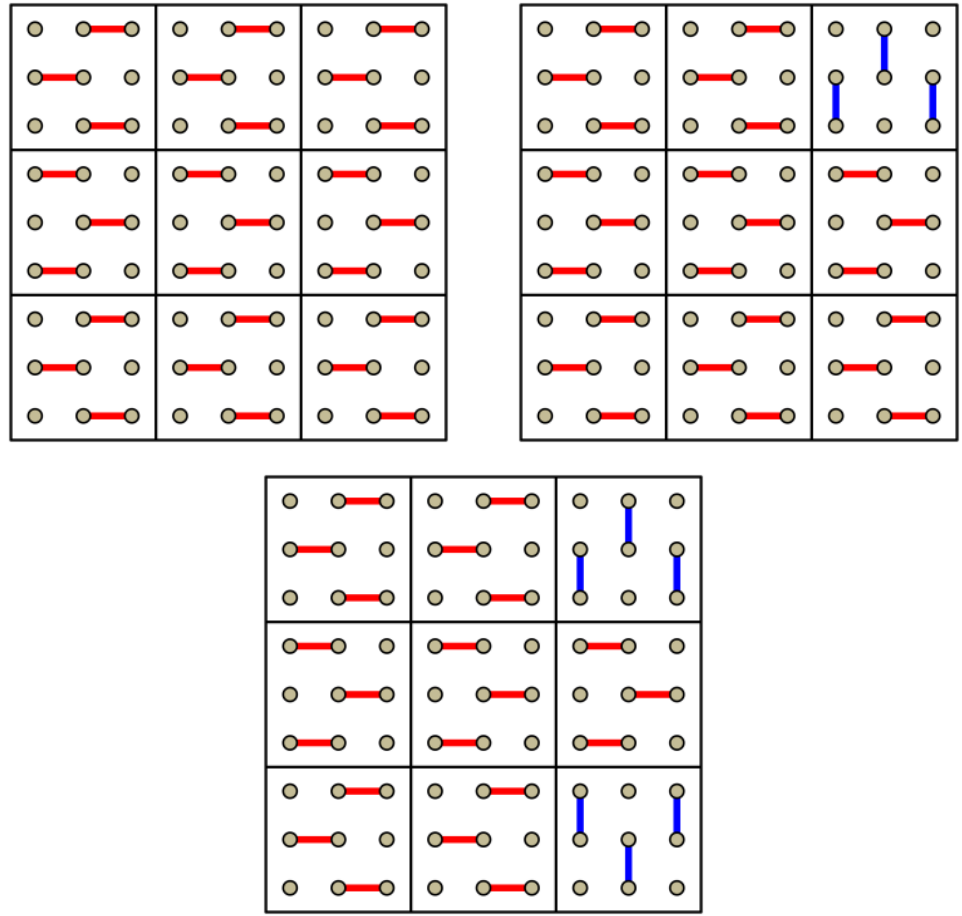

Figure 19. Trivial minimal packings, $n=3 k$ 


$$
\begin{array}{lllll}
\hline 0 & 0 & 0 & 0 & 0 \\
0 & 0 & 0 & 0 & 0 \\
0 & 0 & 0 & 0 & 0 \\
0 & 0 & 0 & 0 & 0 \\
0 & 0 & 0 & 0 & 0
\end{array} \mid \begin{array}{llllllll|}
0 & 0 & 0 & 0 & 0 & 0 & 0 & 0 \\
0 & 0 & 0 & 0 & 0 & 0 & 0 & 0 \\
0 & 0 & 0 & 0 & 0 & 0 & 0 & 0 \\
0 & 0 & 0 & 0 & 0 & 0 & 0 & 0 \\
0 & 0 & 0 & 0 & 0 & 0 & 0 & 0 \\
\hline
\end{array}
$$

Figure 20. Rectangle-free minimal packings, $m=5, n=2+3 k$

$$
\begin{array}{|llllllllll}
0 & 0 & 0 & 0 & 0 & 0 & 0 & 0 & 0 & 0 \\
0 & 0 & 0 & 0 & 0 & 0 & 0 & 0 & 0 & 0 \\
0 & 0 & 0 & 0 & 0 & 0 & 0 & 0 & 0 & 0 \\
0 & 0 & 0 & 0 & 0 & 0 & 0 & 0 & 0 & 0 \\
0 & 0 & 0 & 0 & 0 & 0 & 0 & 0 & 0 & 0 \\
0 & 0 & 0 & 0 & 0 & 0 & 0 & 0 & 0 & 0 \\
0 & 0 & 0 & 0 & 0 & 0 & 0 & 0 & 0 & 0
\end{array}
$$

Figure 21. Rectangle-free packing for $[7 \times 10]$

$$
\begin{array}{|llllllllllllllllll|lllll|}
\hline 0 & 0 & 0 & 0 & 0 & 0 & 0 & 0 & 0 & 0 & 0 & 0 & 0 & 0 & 0 & 0 & 0 & 0 & 0 & 0 & 0 & 0 \\
0 & 0 & 0 & 0 & 0 & 0 & 0 & 0 & 0 & 0 & 0 & 0 & 0 & 0 & 0 & 0 & 0 & 0 & 0 & 0 & 0 & 0 \\
0 & 0 & 0 & 0 & 0 & 0 & 0 & 0 & 0 & 0 & 0 & 0 & 0 & 0 & 0 & 0 & 0 & 0 & 0 & 0 & 0 & 0 \\
0 & 0 & 0 & 0 & 0 & 0 & 0 & 0 & 0 & 0 & 0 & 0 & 0 & 0 & 0 & 0 & 0 & 0 & 0 & 0 & 0 & 0 \\
0 & 0 & 0 & 0 & 0 & 0 & 0 & 0 & 0 & 0 & 0 & 0 & 0 & 0 & 0 & 0 & 0 & 0 & 0 & 0 & 0 & 0 \\
0 & 0 & 0 & 0 & 0 & 0 & 0 & 0 & 0 & 0 & 0 & 0 & 0 & 0 & 0 & 0 & 0 & 0 & 0 & 0 & 0 & 0 \\
0 & 0 & 0 & 0 & 0 & 0 & 0 & 0 & 0 & 0 & 0 & 0 & 0 & 0 & 0 & 0 & 0 & 0 & 0 & 0 & 0 & 0 \\
0 & 0 & 0 & 0 & 0 & 0 & 0 & 0 & 0 & 0 & 0 & 0 & 0 & 0 & 0 & 0 & 0 & 0 & 0 & 0 & 0 & 0 \\
0 & 0 & 0 & 0 & 0 & 0 & 0 & 0 & 0 & 0 & 0 & 0 & 0 & 0 & 0 & 0 & 0 & 0 & 0 & 0 & 0 & 0 \\
0 & 0 & 0 & 0 & 0 & 0 & 0 & 0 & 0 & 0 & 0 & 0 & 0 & 0 & 0 & 0 & 0 & 0 & 0 & 0 & 0 & 0 \\
0 & 0 & 0 & 0 & 0 & 0 & 0 & 0 & 0 & 0 & 0 & 0 & 0 & 0 & 0 & 0 & 0 & 0 & 0 & 0 & 0 & 0 \\
0 & 0 & 0 & 0 & 0 & 0 & 0 & 0 & 0 & 0 & 0 & 0 & 0 & 0 & 0 & 0 & 0 & 0 & 0 & 0 & 0 & 0 \\
0 & 0 & 0 & 0 & 0 & 0 & 0 & 0 & 0 & 0 & 0 & 0 & 0 & 0 & 0 & 0 & 0 & 0 & 0 & 0 & 0 & 0 \\
\hline
\end{array}
$$

Figure 22. Rectangle-free packing for $m=13+3 j, n=7+15 k$ 


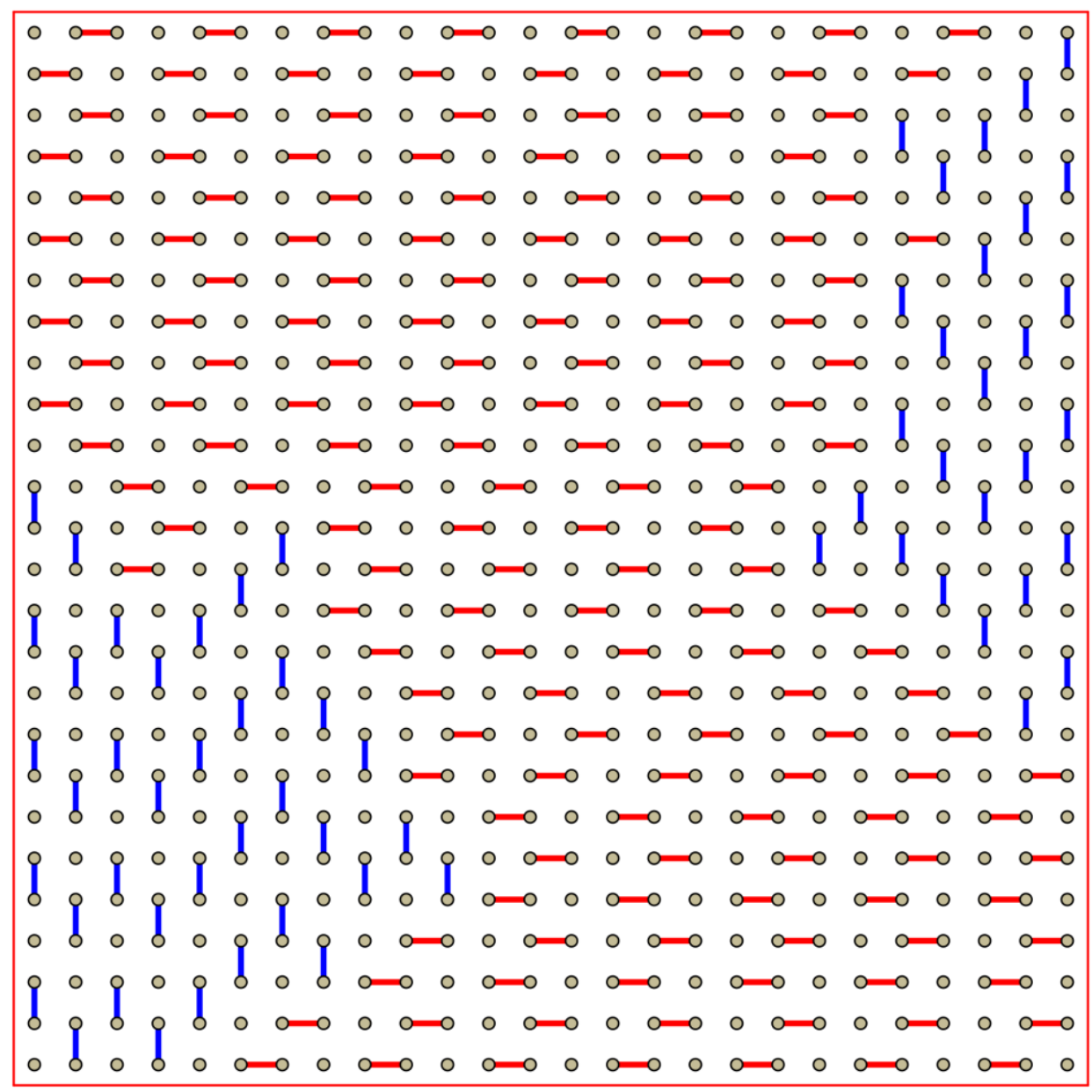

Figure 23. Rectangle-free packing, $d(26)=227$

\section{Basic information for algorithm elaboration}

In this section we use the following notations:

$D_{L}, D_{R}-$ number of dominoes which cover at least one cell in the left (the first), respectively right (the last) column,

$H_{L}, H_{R}-$ number of holes in the left respectively right column.

$T_{L}, T_{R}-\quad$ number of dominoes which touch only dominoes with their left, respectively right edge. (The contact line is one unit for horizontal dominoes and 2 units for vertical dominoes.) 
$M_{L}:=D_{L}+1-H_{L}, M_{R}:=D_{R}+1-H_{R}-$ number of missing holes

$\left(H_{L} \leq D_{L}+1, H_{R} \leq D_{R}+1 \Rightarrow M_{L} \geq 0, M_{R} \geq 0\right)$

$B:=T_{L}+T_{R}+M_{L}+M_{R}-$ number of bad domino constellations,

$$
\begin{gathered}
2(D-H)=T_{L}+T_{R}+D_{L}-H_{L}+D_{R}-H_{R} \\
2(D+1-H)=T_{L}+T_{R}+M_{L}+M_{R}=B .
\end{gathered}
$$

Equality (14) with precision to the notations is equivalent to equality (7) from (Gyárfás et al., 1988). Equality (15) immediately follows from (14). Since $m n=2 D+H$, then $H=m n-2 D$ and

$$
\begin{gathered}
2(D+1-H)=2(3 D+1-m n)=6 D+2(1-m n) \Rightarrow \\
6 D=2(m n-1)+B .
\end{gathered}
$$

Equality (16) is very important. It shows that by minimizing $B$ we minimize $D$.

With a backtracking algorithm we enumerate the domino packings of a $[m \times n]$ rectangle with a given number $D$ of dominoes. We do this by placing dominoes row by row from left to right. In general there are 3 possibilities to continue in a grid cell: empty, horizontal or vertical domino. Therefore the number of paths is greater than $3^{m n / 2}$. Even rather small rectangles cannot be handled as the number of paths is too high.

The new approach considers the known number $B$ of $b a d$ domino constellations. As soon as $(B+1)$ such constellations are reached the current path can be abandoned. For small $B$ this algorithm works very fast. Dependent on the used processor and programming language the enumeration (determination of the number of all packings for a number $D$ of dominoes) for squares up to $[20 \times 20]$ can be done in less than a minute. The status of each cell is described in an oversized array $\mathrm{sq}(\mathrm{x}, \mathrm{y})$ with $0 \leq \mathrm{x} \leq \mathrm{n}+1$ and $0 \leq \mathrm{y} \leq \mathrm{m}+1$, where $\mathrm{x}$ is the column and $\mathrm{y}$ the row, with the following numerical characteristics (Figure 24):

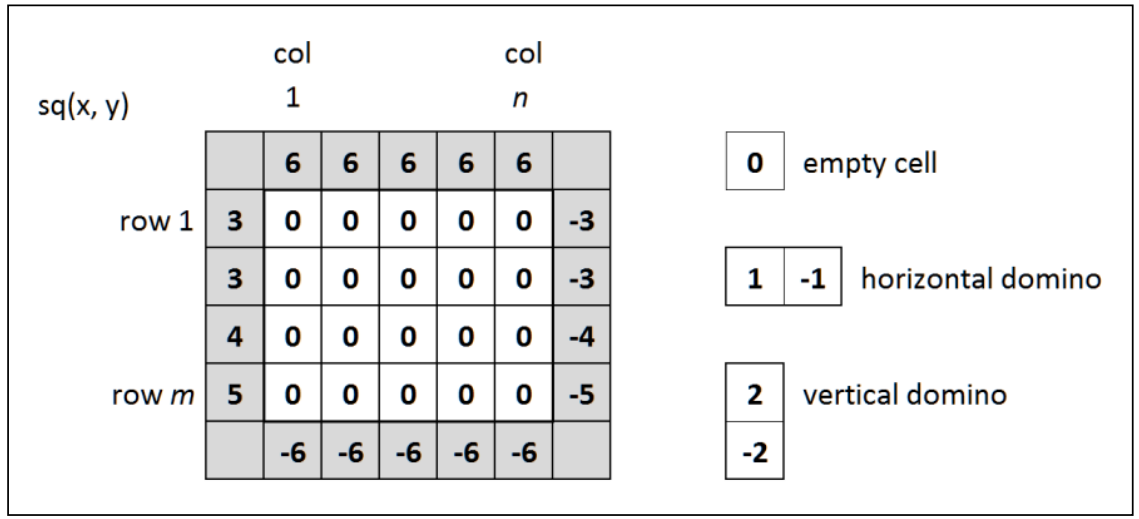

Figure 24. Numerical characteristics 
Horizontal domino

Look at the left neighbor cell of the planned domino (blue)

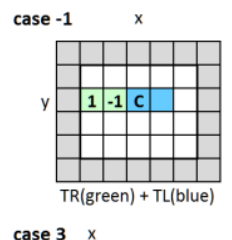
case 2

case -2

case $5 \times$
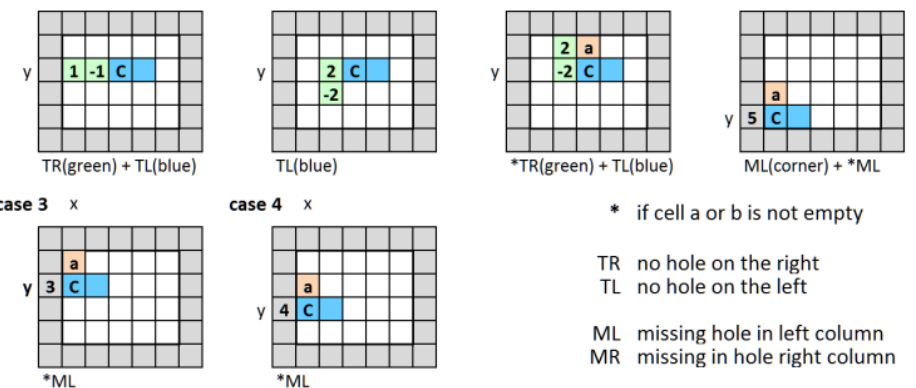

case $4 \times$

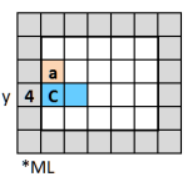

* if cell $a$ or $b$ is not empty

TR no hole on the right

TL no hole on the left

ML missing hole in left column

MR missing in hole right column

Look at the right neighbor cell of the planned domino (blue)

case $-2 x$

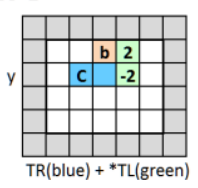

case -5

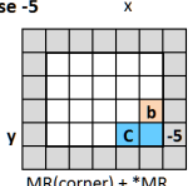

case -3

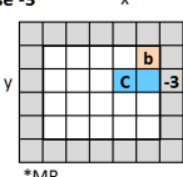

case -4

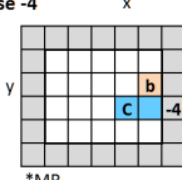

\section{Vertical domino}

Look at the left neighbor cell of the planned domino (blue)

case -1

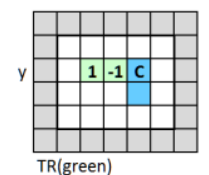

case $3 \times$

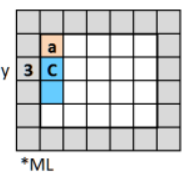

case $2 \quad x$

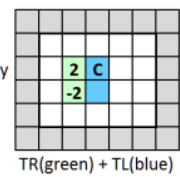

case $4 \times$

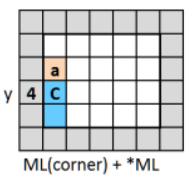

case -

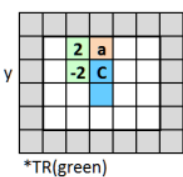

C is the current cell

a is a cell above $c$

b is a cell above the domino but not above $\mathrm{c}$

Look at the right neighbor cell of the planned domino (blue)

case $-2 \quad x$

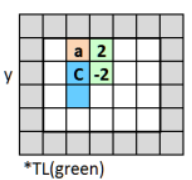

case -3

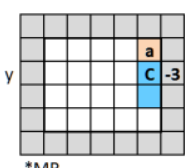

${ }^{*} \mathrm{MR}$

case - 4

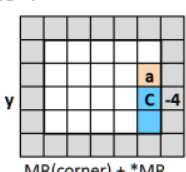

Legend

* if cell a or b is not empty

TR no hole on the right

TL no hole on the left

C is the current cell

ML missing hole in left column

MR missing in hole right column

a is a cell above $c$

b is a cell above the domino but not above $c$

Figure 25. Different cases of domino arrangements 
The source code of the recursive procedure cpos is presented in an easy to read basic pseudo code. All variables are integers and all are public except of $x, y$ and $\mathbf{m B c}$. The main program askes for the values of $m, n$ and $D$, calculates $B$, initializes the array sq() as shown above and calls the procedure by $\operatorname{cpos}(1,1)$. Bad domino constellations were count in BC and compared with $B$. At the end the value of the variable Scnt is the number of different packings. Source code of the recursive procedure cpos ( $x, y$ ) are presented in Appendix.

Different cases for the planned domino as they occur in the procedure are shown in Figure 25.

The most important results obtained with a computer program are summarized in four tables.

Table 1. Number of domin packings in $[n \times n]$-squares with $d(n)=d_{0}(n)$ (including reflections and rotations)

\begin{tabular}{|r|r|r|}
\hline $\mathrm{n}$ & $\mathrm{D}_{0}(\mathrm{n})$ & $\mathrm{P}(\mathrm{n})$ \\
\hline 2 & 2 & 2 \\
\hline 3 & 3 & 4 \\
\hline 4 & 6 & 100 \\
\hline 5 & 9 & 312 \\
\hline 6 & 12 & 14 \\
\hline 7 & 17 & 5020 \\
\hline 8 & 22 & 4804 \\
\hline 9 & 27 & 16 \\
\hline 10 & 34 & 14844 \\
\hline 11 & 41 & 11128 \\
\hline 12 & 48 & 16 \\
\hline 13 & 57 & 7568 \\
\hline 14 & 66 & 4900 \\
\hline 15 & 75 & 16 \\
\hline 16 & 86 & 964 \\
\hline 17 & 97 & 560 \\
\hline
\end{tabular}

\begin{tabular}{|r|r|r|}
\hline $\mathrm{n}$ & $\mathrm{D}_{0}(\mathrm{n})$ & $\mathrm{P}(\mathrm{n})$ \\
\hline 18 & 108 & 16 \\
\hline 19 & 121 & 0 \\
\hline 20 & 134 & 16 \\
\hline 21 & 147 & 16 \\
\hline 22 & 162 & 0 \\
\hline 23 & 177 & 0 \\
\hline 24 & 192 & 16 \\
\hline 25 & 209 & 0 \\
\hline 26 & 226 & 0 \\
\hline 27 & 243 & 16 \\
\hline 28 & 262 & 0 \\
\hline 29 & 281 & 0 \\
\hline 30 & 300 & 16 \\
\hline 31 & 321 & 0 \\
\hline 32 & 342 & 0 \\
\hline 33 & 363 & 16 \\
\hline
\end{tabular}


Table 2. Smallest number $D(m, n)$ of dominoes for which a packing exists

\begin{tabular}{|c|c|c|c|c|c|c|c|c|c|c|c|c|c|c|c|c|c|c|c|c|}
\hline$m \backslash n$ & 14 & 15 & 16 & 17 & 18 & 19 & 20 & 21 & 22 & 23 & 24 & 25 & 26 & 27 & 28 & 29 & 30 & 31 & 32 & 33 \\
\hline 14 & 66 & 70 & 76 & 80 & 84 & 90 & 94 & 98 & 104 & 108 & 112 & 118 & 122 & 126 & 132 & 136 & 140 & 146 & 150 & 154 \\
\hline 15 & & 75 & 80 & 85 & 90 & 95 & 100 & 105 & 110 & 115 & 120 & 125 & 130 & 135 & 140 & 145 & 150 & 155 & 160 & 165 \\
\hline 16 & & & 86 & 92 & 96 & 102 & 108 & 112 & 118 & 124 & 128 & 134 & 140 & 144 & 150 & 156 & 160 & 166 & 172 & 176 \\
\hline 17 & & & & 97 & 102 & 109 & 114 & 119 & 126 & 131 & 136 & 143 & 148 & 153 & 160 & 165 & 170 & 177 & 182 & 187 \\
\hline 18 & & & & & 108 & 114 & 120 & 126 & 132 & 138 & 144 & 150 & 156 & 162 & 168 & 174 & 180 & 186 & 192 & 198 \\
\hline 19 & & & & & & 122 & 128 & 133 & 141 & 147 & 152 & 160 & 166 & 171 & 179 & 185 & 190 & 198 & 204 & 209 \\
\hline 20 & & & & & & & 134 & 140 & 148 & 154 & 160 & 168 & 174 & 180 & 188 & 194 & 200 & 208 & 214 & 220 \\
\hline 21 & & & & & & & & 147 & 154 & 161 & 168 & 175 & 182 & 189 & 196 & 203 & 210 & 217 & 224 & 231 \\
\hline 22 & & & & & & & & & 163 & 170 & 176 & 185 & 192 & 198 & 207 & 214 & 220 & 229 & 236 & 242 \\
\hline 23 & & & & & & & & & & 178 & 184 & 193 & 201 & 207 & 216 & 224 & 230 & 239 & 247 & 253 \\
\hline 24 & & & & & & & & & & & 192 & 200 & 208 & 216 & 224 & 232 & 240 & 248 & 256 & 264 \\
\hline 25 & & & & & & & & & & & & 210 & 218 & 225 & 235 & 243 & 250 & 260 & 268 & 275 \\
\hline 26 & & & & & & & & & & & & & 227 & 234 & 244 & 253 & 260 & 270 & 279 & 286 \\
\hline 27 & & & & & & & & & & & & & & 243 & 252 & 261 & 270 & 279 & 288 & 297 \\
\hline 28 & & & & & & & & & & & & & & & 263 & 272 & 280 & 291 & 300 & 308 \\
\hline 29 & & & & & & & & & & & & & & & & 282 & 290 & 301 & 311 & 319 \\
\hline 30 & & & & & & & & & & & & & & & & & 300 & 310 & 320 & 330 \\
\hline 31 & & & & & & & & & & & & & & & & & & 322 & 332 & 341 \\
\hline 32 & & & & & & & & & & & & & & & & & & & 343 & 352 \\
\hline 33 & & & & & & & & & & & & & & & & & & & & 363 \\
\hline
\end{tabular}

In the coloured cases: $D(m, n)=d_{0}(m, n)+1$

Table 3. Number of minimal domino packings in $[m \times n]$-rectangles with $D_{0}(m, n)$ dominoes (including reflections and rotations)

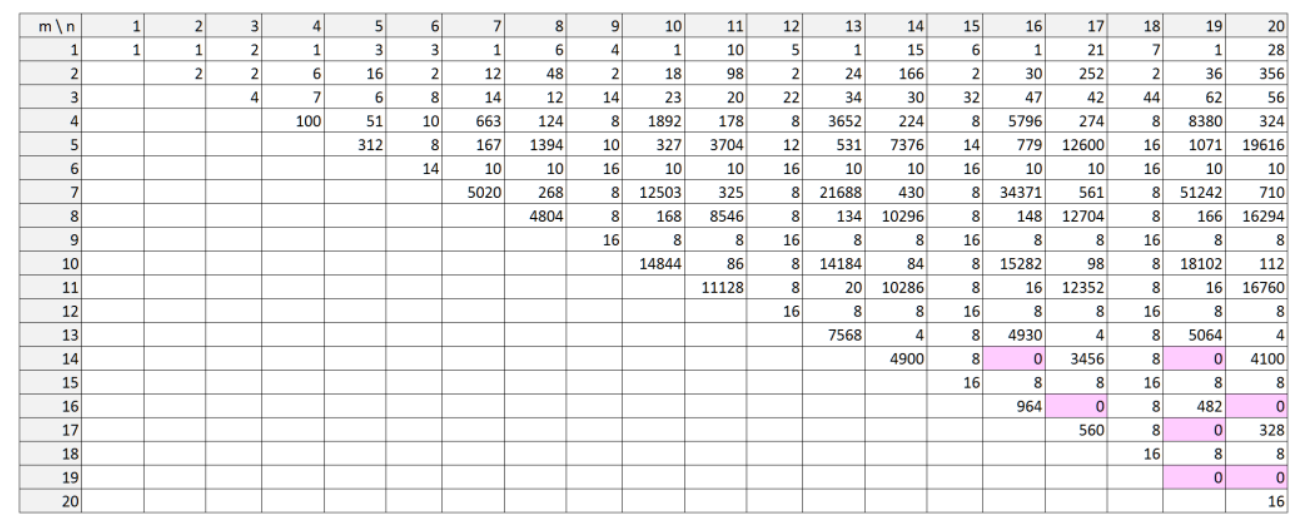




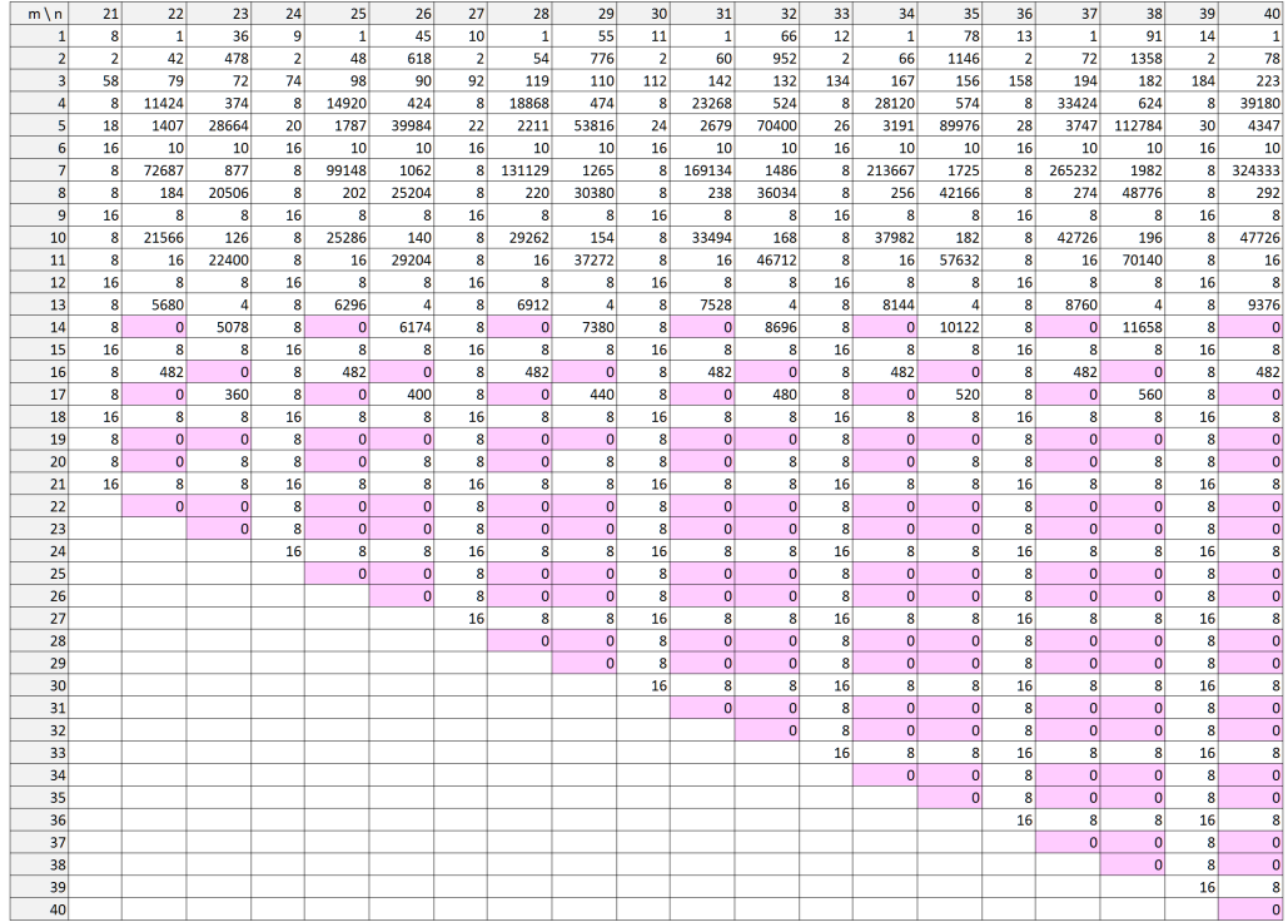

In the coloured cases it was proved by exhaustive computer search that packings with $D_{0}(m, n)$ dominoes do not exist.

\section{Some generalizations}

A natural generalization is the cubic lattice. An estimate

$$
D(m, n, k) \geq\left\lceil\frac{m n k}{3}\right\rceil
$$

can also be used for a three-dimensional rectangles. As in two dimensions, the estimate (17) is sharp if any of the edge lengths is a multiple of 3 . In this case, the minimum packing is obtained by repeating the minimum two-dimensional rectangles in layers. For illustration see Figure 26 with the minimal packing of $[3 \times 4 \times 5]$. More complex packings are shown in Figure 27.

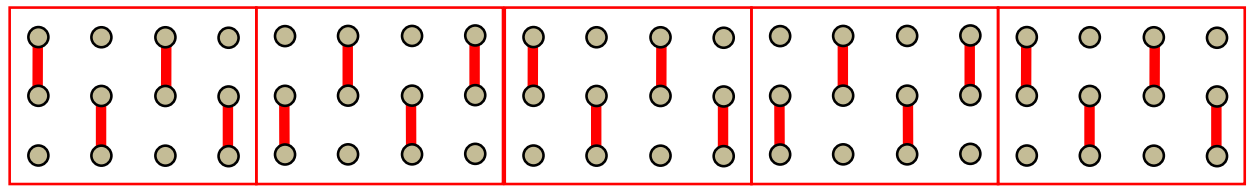

Figure 26. Minimal packing of $[3 \times 4 \times 5]$ in layers 
The results obtained by using a computer program are summarized in Table 4 .

Table 4. Number of minimal packings of $[n \times n \times n]$ cube

\begin{tabular}{|c|c|}
\hline $\boldsymbol{D}(\boldsymbol{n}, \boldsymbol{n}, \boldsymbol{n})$ & Number of packings \\
\hline$D(1,1,1)=0$ & 1 \\
\hline$D(2,2,2)=3$ & 8 \\
\hline$D(3,3,3)=9$ & 6 \\
\hline$D(4,4,4)=22$ & 912 \\
\hline$D(5,5,5)=43$ & 52608 \\
\hline$D(6,6,6)=72$ & 6 \\
\hline
\end{tabular}

From Table 4 we see that $[5 \times 5 \times 5]$ cube is the smallest one for which estimate (17) is no longer sharp. The result $D(4,4,4)=22$ is significant in that the estimate (17) is sharp, but the minimal packing cannot be obtained from the minimal packing of $[4 \times 4]$ rectangles.
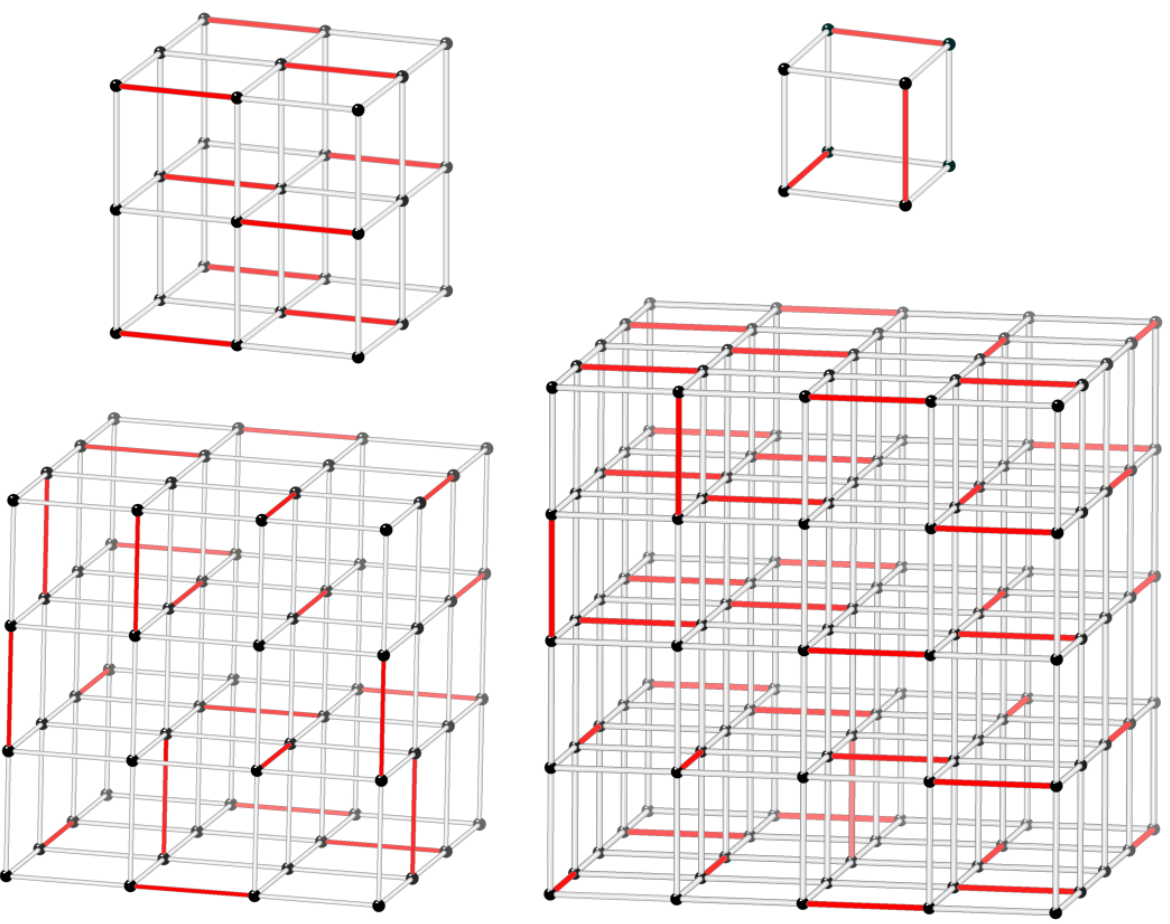

Figure 27. Minimal packings of cubes for $n=2,3,4,5$ 


\section{Conclusions}

The article contains the theorems of pure mathematics, as well as computer-assisted proofs. New progress has been made in solving the domino exclusion problem, including a deeper understanding of the structure of minimal packings. Proof (or disproof) of the hypothesis formulated in the Remark 4 could be a natural continuation of this study.

\section{References}

Alanko, S., Crevals, S., Isopoussu, A., Östergård, P., Pettersson, W. (2011). Computing the Domination Number of Grid Graphs, The electronic journal of combinatorics 18. Available at https://www.academia.edu/1275986/Computing the Domination Number of Gri d Graphs

Golomb, S. W. (1994). Polyominoes: Puzzles, Patterns, Problems and Packings, Princeton, University Press, NJ. (First edition: Polyominoes, New York: Scribners Sons, 1965)

Gyárfás, A., Lehel, J., Tuza, Z. (1988). Clumsy Packing of Dominoes, Discrete Mathematics, 71, 33 - 46. Available at: https://www.sciencedirect.com/science/article/pii/0012365X88900283

Kagey P. (2019), Minimum number of dominoes on an n X n chessboard to prevent placement of another domino, Mathematics Stack Exchange,

https://math. stackexchange.com/questions/3191721/minimum-number-ofdominoes-on-an-n-times-n-chessboard-to-prevent-placement-of

Korte, B., Vygen, J. (2018). Combinatorial Optimization. Theory and Algorithms, Sixth edition, Springer-Verlag GmbH, Germany.

Shepard R. L. (2017). Minimum number of dominoes on an $n X n$ chessboard to prevent placement of another domino, The On-line Encyclopedia of Integer Sequences, http: //oeis.org/search?q=A280984

Received July 30, 2020, revised October 2, 2020, accepted October 22, 2020 
Appendix: Source code of the recursive procedure cpos $(x, y)$

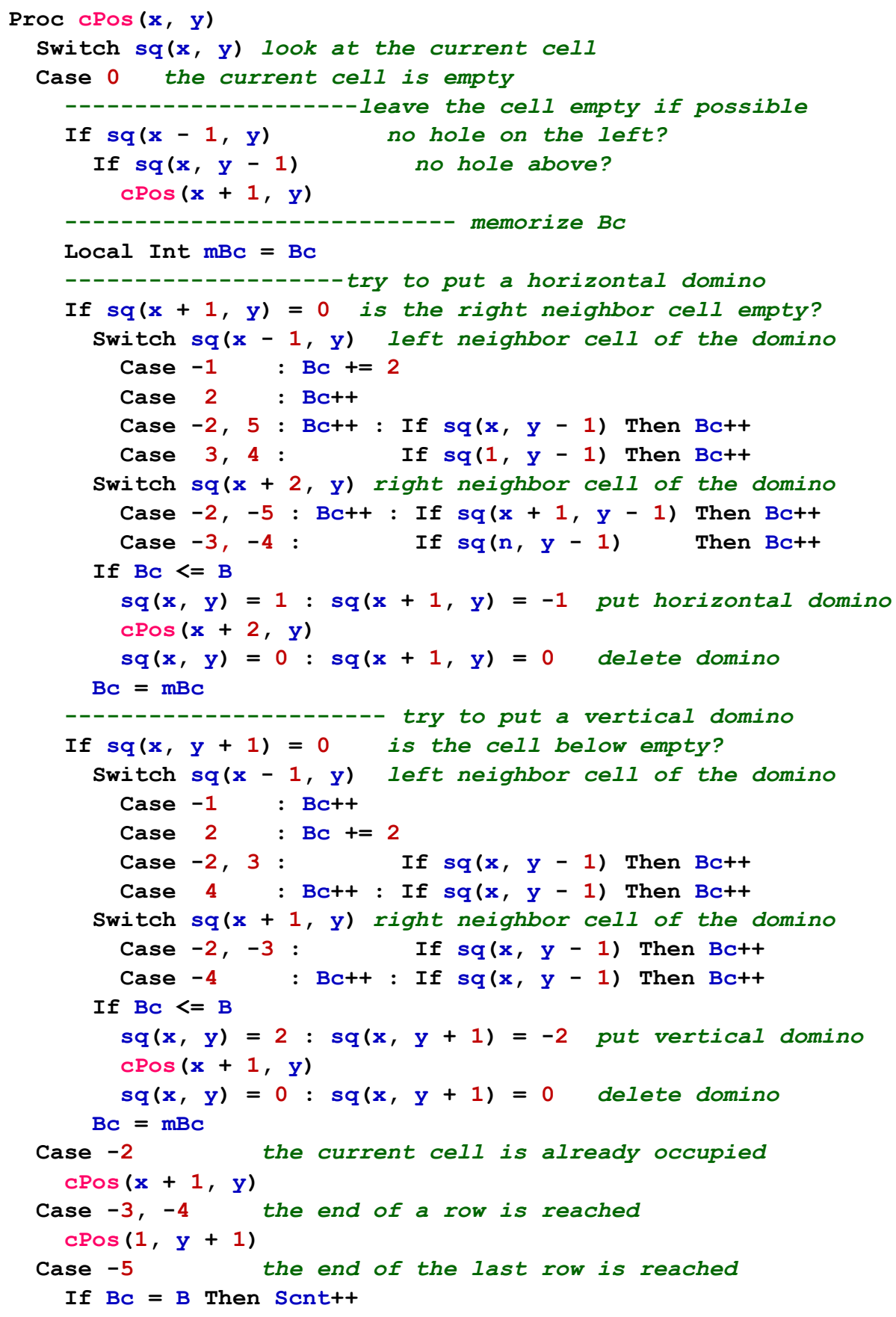

